\title{
Elliptical stretch as a cause of side branch ostial compromise after main vessel stenting in coronary bifurcations: New insights from numerical analysis
}

\author{
Dobrin Iotkov Vassilev ${ }^{1}$, Ghassan S. Kassab ${ }^{2}$, Carlos Collet ${ }^{3}$, \\ Juan Luis Gutiérrez-Chico ${ }^{4}$, Gianluca Rigatelli ${ }^{5}$, Robert J. Gil ${ }^{6,7}$, Patrick W. Serruys ${ }^{8,9}$ \\ ${ }^{1}$ Cardiology Clinic, "Alexandrovska” University Hospital, Medical University of Sofia, Bulgaria \\ ${ }^{2}$ California Medical Innovations Institute, San Diego, California \\ ${ }^{3}$ Department of Cardiology, University of Amsterdam, Netherlands \\ ${ }^{4}$ La Charité, Campus Benjamin Franklin, Berlin, Germany \\ ${ }^{5}$ Cardiovascular Diagnosis and Endoluminal Interventions, Director Section of Adult Congenital \\ Heart Interventions Rovigo General Hospital, Rovigo, Italy \\ ${ }^{6}$ Mossakowski Medical Research Centre, Polish Academy of Sciences, Warsaw, Poland \\ ${ }^{7}$ Department of Invasive Cardiology, Central Clinical Hospital of \\ the Ministry of the Interior, Warsaw, Poland \\ ${ }^{8}$ Erasmus MC, Imperial College London, United Kingdom \\ ${ }^{9}$ Thorax Center, Cardiovascular Science Division NHLI, Amsterdam, Netherlands
}

\begin{abstract}
Background: The side branch (SB) compromise after main vessel (MV) stenting remains a significant problem in coronary bifurcation treatment. Currently the two major hypotheses for the mechanism of $S B$ compromise are carina shift from MV into the SB and plaque shift into the ostium of side vessel. It is proposed herein, SB ostial deformation leading to reshaping of the ostium from circle to ellipse is a third possible mechanism. In the current study, the theoretical effects and correlation of ostial deformation with fractional flow reserve (FFR) is explored.

Methods: Based on angiographic measurements and theoretical analysis formulas, three different $S B$ ostial areas using circular ostial shape assumption and elliptical ostial shape assumption were calculated. Three different types of ostial areas with FFR values after MV stenting in 49 patients from the FIESTA registry were compared and analyzed.

Results: It was found that there is significant overestimation of stenosis severity when estimated by the circle formula, than with the ellipse formula - ASc vs. ASds with $25 \% \pm 13 \%, p<0.001, A S c$ vs. ASmld with $9 \% \pm 10 \%, p<0.001$. The elliptical shape assumptions provide more accurate ostial area stenosis, which correlates better with FFR. This finding is more significant in less severe stenosis $(<70 \%$ area stenosis) than in a more severe one.

Conclusions: A third possible mechanism of SB compromise after MV stenting of coronary bifurcation stenosis is elliptical ostial deformation at the ostium of SBs. The ostial area, calculated based on elliptical assumption correlates better with FFR, than area stenosis calculated with the traditional circular formula. (Cardiol J 2020; 27, 5: 507-517)
\end{abstract}

Key words: coronary bifurcation, side branch ostium compromise, elliptical ostial stretching

Editorial p. 474

Address for correspondence: Dobrin Vassilev, MD, PhD, "Alexandrovska” University Hospital, Medical University, “St. George Sofiiski” Str. 1, 1431 Sofia, Bulgaria, tel: +35 929230467, e-mail: dobrinv@gmail.com 


\section{Introduction}

There have been major improvements in treatment of coronary bifurcations in recent years [1]. Nowadays, there is much more known about stent characteristics which are important in achieving good procedural results. With the advent of drug-eluting stents (DES) the problem of restenosis was largely reduced. However, the main reason which makes coronary bifurcation so difficult to treat still persists - namely, side branch compromise which is the appearance of high grade ostial stenosis at the ostium of the side branch (SB), limiting vessel inflow after implantation of a stent in the main vessel (MV). In the most severe form of SB compromise, the vessel can occlude leading to a different size periprocedural myocardial infarction with different prognostic implications, depending on amount of subtended myocardium by the SB. There is still uncertainty about the mechanisms of SB compromise after MV stenting in coronary bifurcation lesions. There are currently two major hypotheses: 1) Plaque shift from MV into the SB and 2) Carina shift due to pushing of carina tip into the ostium of side vessel [1]. Based on theory, phantom elastic models and then on angiographic analysis from the patient cohort, demonstrated herein, that carina displacement is probably the most important mechanism for SB stenosis [2-4]. Besides possible plaque shift (from proximal MV to $\mathrm{SB}$ ostium and plaque redistribution of $\mathrm{SB}$ plaque in a circumferential direction), there is however, another potential mechanism; i.e., ostial deformation resulting in reshaping of side vessel ostium from ostial circle initially to ostial ellipse after stenting [3]. These potential changes were recently reported in human coronary bifurcation after stenting of MV [5, 6]. Thus, the present study proposed this as a third possible mechanism for SB compromise, which can operate in conjunction with carina displacement and plaque shifting. Here, the theoretical effects are explored and a correlation of these possible deformational effects with fractional flow reserve (FFR), serve as a current standard for assessment of stenosis severity [7].

\section{Methods}

A theoretical analysis was performed on the potential changes at the SB ostium after MV stenting. Formulas were derived for minimal lumen diameter at SB opening after stenting based on an assumed elliptical stretch, with constant vessel circumference irrespective of vessel deforma- tions. An angiographic analysis was performed to measure minimal lumen diameter and reference diameters of the bifurcation region from a patient cohort, with FFR during coronary bifurcation intervention being simultaneously measured.

Based on angiographic measurements and using formulas from theoretical analysis, three different SB ostial areas were calculated which were then compared with FFR values after MV stenting. First, a circular ostial shape at SB ostium was assumed after stenting using the formula: $\mathbf{A}_{\mathrm{c}}=\pi . \mathbf{d s}^{2} / \mathbf{4}$, where ds is reference side branch vessel diameter as measured from quantitative coronary assessment (QCA). This is a standard calculation used in two-dimensional QCA software packages. Second, we assumed elliptical ostial shape of SB ostium after MV stenting. The calculation of the ostial area uses the ellipse area formula $\mathrm{A}=\pi$.a.b, where $a$ is the ostial minimal lumen diameter as a minor semi axis. This minor semi axis "a" equivalent of SB ostial minimal lumen diameter, as measured from standard QCA. The major semi axis $(b)$ was calculated using formulas 3'-12' (see below), replacing $\mathrm{k}$ (stretching coefficient) with its equivalent (ds/2)/ $a$ (where $d s$ is $\mathrm{SB}$ reference diameter and a is ostial elliptic minor axis after stent placement in the MV). The SB diameter, $d s$, was taken as a reference in those calculations. The respective area stenosis was calculated as ASds $=(1-\mathrm{Ae} 1 / \mathrm{Asb}) \times 100$, where ASds is ostial elliptic area stenosis of the SB, Ae1 - SB calculated ostial area, Asb - reference SB vessel area (calculated based on vessel diameter $1 \mathrm{~mm}$ distal from the end of visually diseased end of plaque segment). For the third calculation of SB ostial area after stenting, the same assumptions and formulas were used as in the second, but as a reference diameter instead of SB reference diameter the SB ostial minimal lumen diameter before stenting was used (i.e. this is the minimal lumen diameter before stenting, as measured from $\mathrm{QCA})$. The corresponding area stenosis was labeled ASmld $=(1-A e 2 / A s b) \times 100$, where ASmld is ostial area stenosis (in percentages), Ae2 - ostial SB area calculated according to the above assumptions, Asb - as above.

All three calculated areas were correlated with FFR measured in SB after stenting to determine functional significance of ostial stenosis. In theory, the flow through SB ostium should be proportional to its cross-sectional area and the subtended myocardium. Hence, a better estimate of real cross-sectional area of side vessel opening should correlate better with FFR. 


\section{Theoretical analysis}

A model of bifurcation with normal opening of proximal MV was assumed, with a diameter dp at the point of distal MV and SB divergence. The SB has a circular opening in a plane perpendicular to the plane of bifurcation. The diameters of distal $\mathrm{MV}$ and SB are denoted as $\mathrm{dm}$ and ds, accordingly. At the point of connection of the three tubes there is a beveling region with a length equal to SB tube diameter. The vessels are assumed to be deformable straight tubes at the region of interest. No other assumptions were made regarding the model (Fig. 1A). After stent placement in MV across the SB, the stent stretches a bevel region of bifurcation causing "squeezing" SB ostium and ellipse formation at the opening [4]. Those changes were described in experimental elastic model by our group and currently reported to occur in human patients with optical coherence tomography observations $[5,6]$.

The SB minimal lumen diameter was calculated, SB ostial area and respective derived parameters. It was assumed that after stenting MV, the SB stretched to ellipse geometry at the ostium. The short axis of a newly formed ellipse was parallel with SB long axis and the short axiswas in a perpendicular direction (Fig. 2). This new elliptic short axis is a minimal lumen diameter for SB ostium and the area of ellipse relative area of reference cross-section of the SB is a lumen area stenosis of the ostium. If the vessel wall is inelastic and the vessel perimeter remains constant during simple deformations without circumferential stretching forces, the minimal (short) ellipse diameter, a, is determined from the extent of stretching (lateral increase) of SB vessel major axis $b$. The major axis can be expressed as a multiple of SB reference diameter: a' $=\mathrm{k} . \mathrm{ds} / 2$, where ds is the SB diameter (measured from QCA side branch reference vessel diameter) and $\mathrm{k}$ is the stretch coefficient. The stretch coefficient can vary to two maxima $\mathrm{k} 1=\mathrm{dm} / \mathrm{ds}$ or $\mathrm{k} 2=\mathrm{dp} / \mathrm{ds}(\mathrm{dm}-\mathrm{MV}$ distal diameter, dp - proximal MV diameter), depending on the choice of stent diameter. According to the above assumption of constant vessel perimeter and knowing the extent of ostial stretch (expressed as the value of $\mathrm{k}$ ), the minimal diameter at the ostium was calculated. Using elementary integration methods, the perimeter $\mathrm{S}(\mathrm{a}, \mathrm{b})$ of the ellipse defined by $(x / a)+(y / b)=1$, is given by:

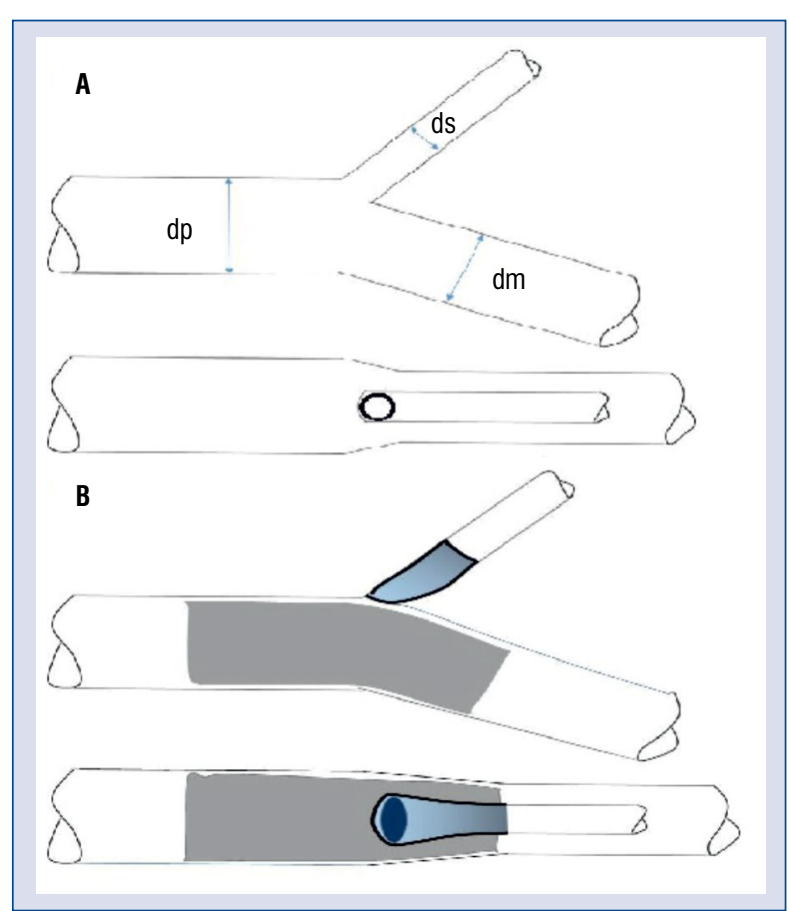

Figure 1. A. Model of bifurcation before stent placement; dp - proximal main vessel diameter; dm distal main vessel diameter; ds - side branch vessel diameter; B. Lateral and axial views of bifurcation region after stent placement. The stent (gray rectangle) pushes the carina to the side branch ostium and causes widening and stretching of beveling region, which in fact stretches side branch ostium in a perpendicular direction of the main vessel axis. This leads to an elliptical shape of branch ostium.

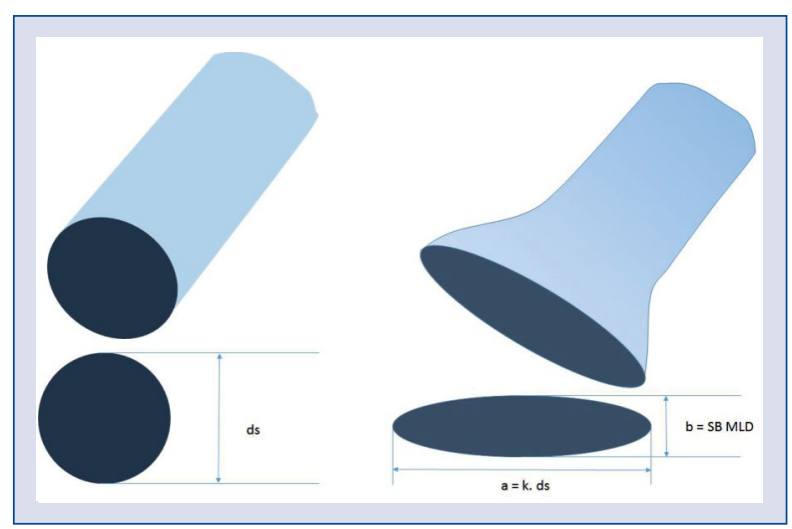

Figure 2. Idealized view of side branch (SB) ostium before and after stent placement; i.e., before and after stretching. $a$ and $b$ are major and minor axes of newly formed ellipse; ds - side branch reference diameter; $\mathrm{k}$ - stretching coefficient, being the diameter of ellipse after transformation from a circle; MLD - minimal lumen diameter. 
$\mathrm{S}(\mathrm{a}, \mathrm{b})=4 \mathrm{aE}\left(\mathrm{e}^{2}\right)$, where $\mathrm{e}=\sqrt{\mathrm{a}^{2}-\mathrm{b}^{2}} / \mathrm{a}$

where E, the ellipse eccentricity, is given by:

$\left.\mathrm{E}(\mathrm{x})=\int_{0}^{\pi / 2} 1-\mathrm{x} \cdot \sin ^{2} \theta\right)^{1 / 2} \mathrm{~d} \theta$

$\mathrm{E}$ is an elliptic integral of the second kind, which can be computed with numeric integration or by approximations. The problem with equation (1) is that $\mathrm{E}(\mathrm{x})$ is a transcendental function and its evaluation through an infinite series or fractions is computationally inefficient. Therefore, it was decided that some approximation formulas would be used, giving in the ellipse perimeter computation less than $1 \%$ error in comparison with exact computation [8-15]. For each formula below (denoted by numerical equation), we give a derivative formula (denoted by prime) for short axis semi diameter, based on values of SB diameter and stretch coefficient as independent variables.

$\mathrm{P}=2 \pi\{[\mathrm{p} .(\mathrm{a}+\mathrm{b}) / 2]+[(1-\mathrm{p})$.

$\sqrt{ }(\mathrm{a} . \mathrm{b})]\}$, with $\mathrm{p}=3 / 2 \quad$ Optimized Peano (3)

$\mathrm{b}=\mathrm{ds} / 9 .\left\{6-7 \mathrm{k} / 2+\left(2 . \sqrt{ }\left(3 \mathrm{k}-2 \mathrm{k}^{2}\right)\right\}\right.$

$\mathrm{P}=2 \pi\left\{\left(\mathrm{a}^{3 / 2}+\mathrm{b}^{3 / 2}\right) / 2\right\}^{2 / 3}$ Muir (4)

$\mathrm{b}=\mathrm{ds} / 2 .\left\{2-\mathrm{k}^{3 / 2}\right\}^{2 / 3}$

$\mathrm{P}=\pi\{3 .(\mathrm{a}+\mathrm{b})-\sqrt{ }[(\mathrm{a}+3 \mathrm{~b})(3 \mathrm{a}+\mathrm{b})]\}$ Ramanujan (5)

$\mathrm{b}=\mathrm{ds} / 6 .\left\{3-2 \mathrm{k}+\sqrt{ }\left(3+6 \mathrm{k}-5 \mathrm{k}^{2}\right)\right\}$

$\mathrm{P}=2 \cdot \sqrt{ }\left[\pi^{2} \mathrm{a} \cdot \mathrm{b}+4 \cdot(\mathrm{a}-\mathrm{b})^{2}\right]$

$\mathrm{b}=\mathrm{ds} / 16 .\left\{\mathrm{k} \cdot\left(8-\pi^{2}\right)+\pi \sqrt{\left[\mathrm{k}^{2}\left(\pi^{2}-16\right)+16\right]}\right.$

$\mathrm{P}=4 .\left(\mathrm{a}^{\mathrm{s}}+\mathrm{b}^{\mathrm{s}}\right)^{1 / \mathrm{s}}, \mathrm{s}=\ln 2 / \ln (\pi / 2)=$

$=1.53492853566$

Marthens (7)

$\mathrm{b}=\mathrm{ds} / 2 .\left[(\pi / 2)^{\mathrm{s}}-\mathrm{k}^{\mathrm{s}}\right]^{1 / \mathrm{s}}$

$\mathrm{P}=4 \mathrm{a}+\left\{2 \cdot(\pi-2) \cdot \mathrm{a} \cdot(\mathrm{b} / \mathrm{a})^{1.456}\right\}$

Rivera (8)

$\mathrm{b}=\mathrm{ds} / 2 . \mathrm{k} \cdot\left\{[(\pi-2 \mathrm{k}) /(\mathrm{k} .(\pi-2))]^{0.6868}\right\}$

$\mathrm{P}=4\left\{\left[\pi \cdot \mathrm{a} \cdot \mathrm{b}+(\mathrm{a}-\mathrm{b})^{2}\right] /[\mathrm{a}+\mathrm{b}]\right\}$ Rivera-Sykora $(9)$

$\mathrm{b}=\mathrm{ds} / 8\{\pi+4 \mathrm{k}-2 \pi \mathrm{k}+\sqrt{ }[(4 \mathrm{k} \pi .(\mathrm{k}-1)$

$\left.\left.(\pi-4))+\pi^{2}\right]\right\}$

$\mathrm{P}=2 . \pi \sqrt{ }\left\{\left[\mathrm{w} .\left(\mathrm{a}^{2}+\mathrm{b}^{2}\right) / 2\right]+[(1-\mathrm{w})(\mathrm{ab})]\right\}$ QO1 (10)

$\mathrm{b}=\mathrm{ds} / 2\left\{[(\mathrm{w}-1) \cdot \mathrm{k}]+\sqrt{\left.\left[\mathrm{k}^{2}(1-2 \mathrm{w})+2 \mathrm{w}\right]\right\}}\right.$

The parameter $\mathrm{w}$ can be optimized, giving the best result at $\mathrm{w}=1.007$.

$\mathrm{P}=\pi \sqrt{ }\left\{\left[2\left(\mathrm{a}^{2}+\mathrm{b}^{2}\right)\right]-\left[(\mathrm{a}-\mathrm{b})^{2} / \mathrm{D}\right]\right\}$

$\mathrm{b}=\mathrm{ds} / 2\left\{\left[-\mathrm{k}+2 \sqrt{ }\left(\mathrm{D}\left(\mathrm{k}^{2}(1-\mathrm{D})+2 \mathrm{D}-1\right)\right)\right] /(2 \mathrm{D}-1)\left(11^{\prime}\right)\right.$

The parameter $\mathrm{D}$ gives optimal results with values between 2 and $3(\mathrm{D}=2.5$ for present analysis).

$\mathrm{P}=\varpi \cdot\left\{(\mathrm{a}+\mathrm{b}) / 2+\sqrt{ }\left[\left(\mathrm{a}^{2}+\mathrm{b}^{2}\right) / 2\right]\right\} \quad \mathrm{QO} 3$

$\mathrm{b}=\mathrm{ds} / 2[-4+\mathrm{k}+4 \sqrt{ }(2-\mathrm{k})]$
All the above formulas at $\mathrm{k}=1$ (circular shape) reduce to a simple formula for circle perimeter with a radius equal to ds $/ 2$. There are certain limits of $\mathrm{k}$ values - in most of the cases it cannot be $>1.6$. Therefore, being tested were the results for $\mathrm{k}$ varying between 1.1 and 1.5. The ostial elliptic area, $\mathrm{A}_{\mathrm{x}}=\pi$.ds. $\mathrm{b}$ and $\mathrm{SB}$ reference circular area $\left(\mathrm{A}_{\mathrm{c}}=\pi \cdot \mathrm{ds}^{2} / 4\right)$, were compared to determine possible ostial percent area stenosis, AS $=\left(1-\mathrm{A}_{\mathrm{x}} / \mathrm{A}_{\mathrm{c}}\right)$ $\times 100$. The ostial percent diameter stenosis was calculated as DS $=(1-b / d s) \times 100$. The derived eccentricity of the ostium of SB was expressed as $\mathrm{e}=\mathrm{a} / \mathrm{b}$, where $\mathrm{a}$ and $\mathrm{b}$ are major and minor semi axes, respectively.

For practical calculations (see Results section) formula 5 (Ramanujan) was used for calculation of the area stenosis. For calculation of area stenosis after stenting numerical values were used from QCA as follows: for ASds minimal lumen diameter after stenting divided to SB reference diameter to derive parameter $\mathrm{k}=\mathrm{SBMLD} / \mathrm{SBRVD}$ were used; then this value was used to calculate parameter $b$ in formula 5 , and then the ostial area was calculated as Ads $=\pi$.SBMLD.b For calculation of ASmld (see Results section) for calculation of parameter $\mathrm{k}$ the SBMLD before stenting was used: $\mathrm{k}=$ $\mathrm{SBMLD}_{\text {afterstent }} / \mathrm{SBMLD}_{\text {beforestent }}$, then the parameter $\mathrm{b}$ was calculated as described and the ostial area was calculated in the same way: Amld $=\pi$.SBMLD.b According area stenosis is derived by dividing ostial area by SB reference area $\mathrm{A}_{c}=\pi \cdot \mathrm{ds}^{2} / 4$.

\section{Angiographic analysis}

Quantitative angiographic analyses were performed using commercially available software (Medis QCA version 5.0, Leiden, the Netherlands; Dicom Works version 3.1.5b, Paris). Catheter calibration was used in all cases. Bifurcation lesions were classified according to the visual Medina classification using an index of 1 for stenoses greater than $50 \%$ and an index of 0 for no stenosis. The changes of SB percentage diameter stenosis (SB\%DS) before procedure, after stenting and at the end of percutaneous coronary intervention (PCI) were assessed. SB reference diameter, as well as minimal lumen diameters were measured before and after stenting, after giving $100 \mu \mathrm{g}$ nitroglycerin intracoronary.

\section{Procedures}

Patients from the FIESTA registry were analyzed, which was a continuation of the FIESTA 
study (Ffr vs. IcEcgSTA) [15]. Briefly, patients with stable or unstable angina were included. The inclusion criterion were angiographic bifurcation lesions in a native coronary artery with a diameter $\geq 2.5 \mathrm{~mm}$ and $\leq 4.5 \mathrm{~mm}$ and SB diameter $\geq 2.0 \mathrm{~mm}$. Patients with ST-segment elevation myocardial infarction and those with non-cardiac co-morbidity conditions with a life expectancy of less than one year were excluded. PCI was performed according to current guidelines. Provisional stenting was the default strategy in all patients. Two guidewires were inserted into both distal MB and SB. Initial FFR and post-stenting FFR was performed using PrimeWire or PrimeWire Prestige (Volcano Corp., USA). For all FFR measurements, intracoronary adenosine was given in increasing doses of $60 \mu \mathrm{g}, 120 \mu \mathrm{g}$, and $240 \mu \mathrm{g}$. The minimum value of FFR measurements was taken for analysis. Pre-dilatation of MV was mandatory. The SB balloon predilatation was left to operator discretion, regardless of the initial FFR values. All patients received double antiplatelet therapy with ADPantagonist and acetylsalicylic acid for at least 12 months.

\section{Statistics}

Continuous data are presented as mean \pm standard deviation. Differences between groups were examined with paired or unpaired t-tests as appropriate, with normal distributions. If the distribution was not normal, the Wilcoxon sign-ranked test and Mann-Whitney U-tests was performed. Analysis of variance (ANOVA) was used for multiple comparisons of data, when parameters were distributed normally. Otherwise, the Kruskall-Wallis test was performed. Correlation analysis as well as univariate regression analysis were performed to identify associates of "significant" cut-off value of FFR. For purposes of current analysis, test 0.80 were made cut-off values for FFR [7]. All univariate predictors with $\mathrm{p}<0.1$ were included in a multivariate model. Chi-square tests were applied for qualitative data. For determining of cut-off values for continuous parameters a receiver-operation curve analysis was performed, determining sensitivity and specificity of a given value. A p $<0.05$ was accepted as statistical significance.

\section{Results}

\section{Theoretical analysis}

For the theoretical analysis that diameters of main branch vessel varying between the 2.5-4.0 $\mathrm{mm}$ range were assumed and side branch diameter varied between $1.5-3.0 \mathrm{~mm}$. Figures 3 and 4 present the calculated percentage diameter stenosis and area stenosis based on elliptical ostial shape assumption and circular ostial shape assumption.

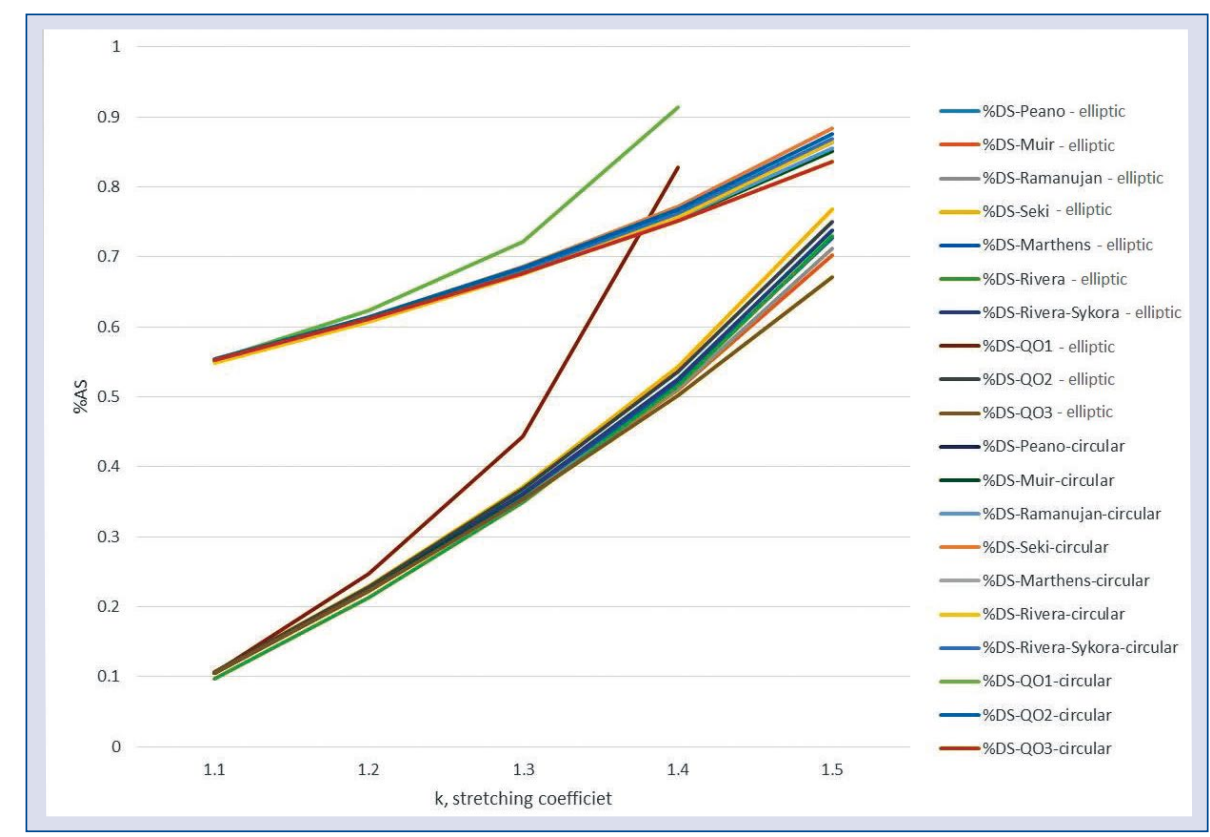

Figure 3. Percentage diameter stenosis change depending on coefficient of stretch. The upper curves represent \%AS if ostial circular shape is assumed. There is considerable overestimation of stenosis severity with circular shape assumption. 


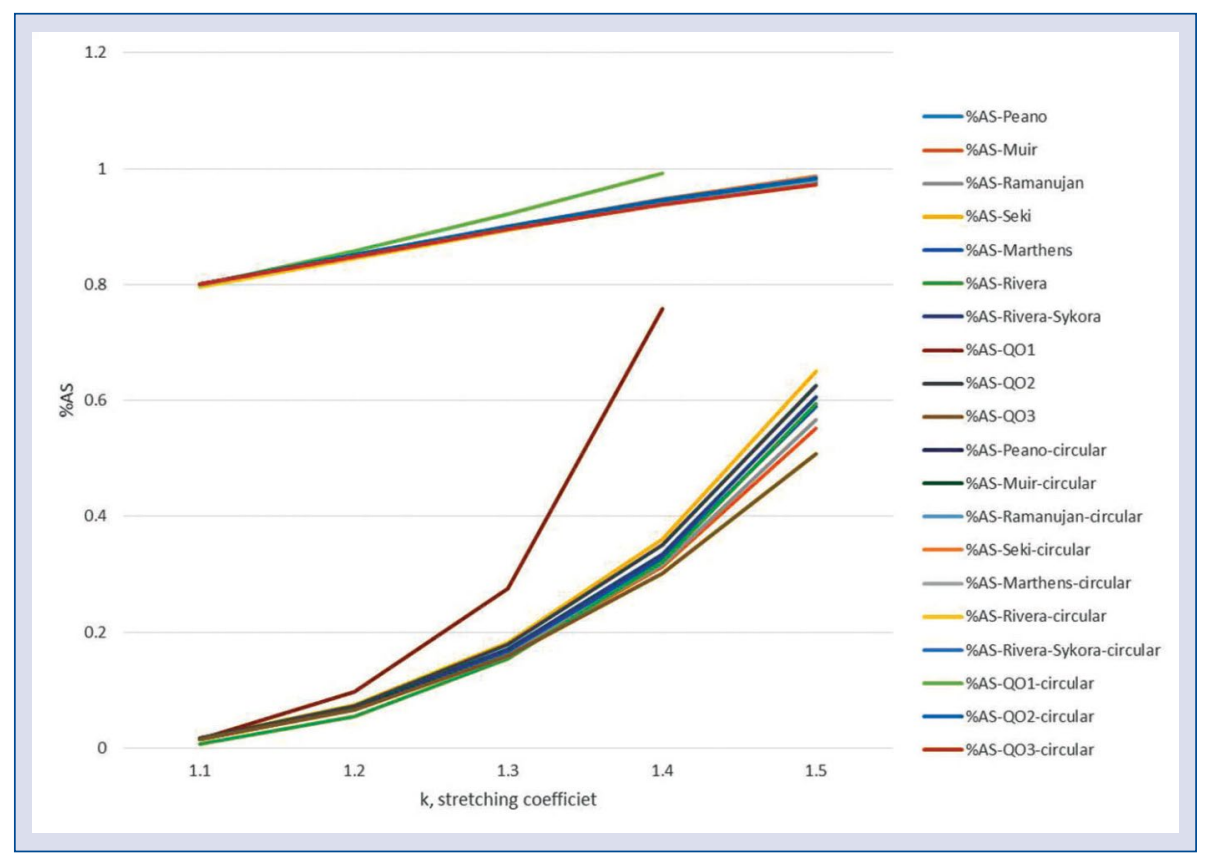

Figure 4. Percentage area stenosis change depending on coefficient of stretch. The upper curves represent \%AS if an ostial circular shape is assumed.

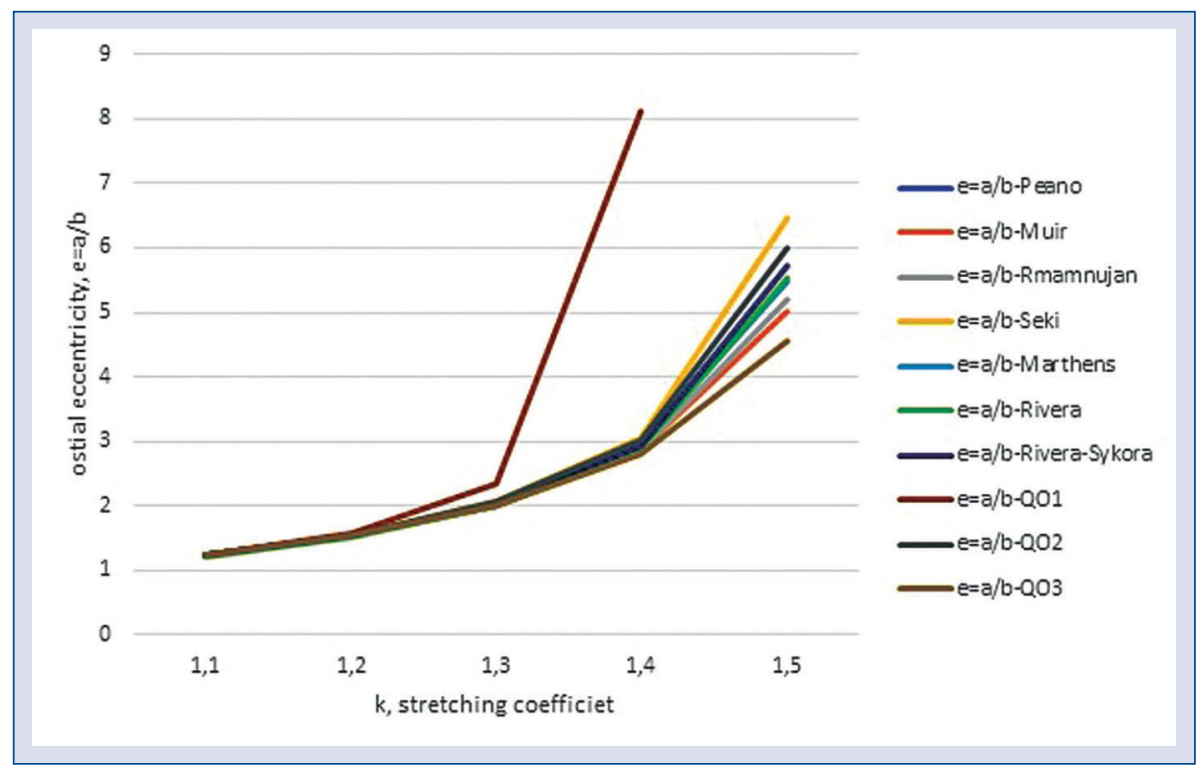

Figure 5. Ostium eccentricity. There is a striking increase when stretch is greater than 1.4.

With an increase in stretching coefficient, there is a reduction of overestimation (based on QCA data) of stenosis severity if circular shapes are assumed. The overestimation is larger for percentage diameter stenosis than for area stenosis (between $80 \%$ and $35 \%$ in absolute value), however, this means a significant difference in cross-sectional area of SB ostium. In high grade stenoses (> 90\% diameter stenosis the differences between elliptical and circular calculated area stenoses were small. The quadratic optimization (Eqn. 1) gives larger deviations than the other formulas giving closer to circular approximation values for \%DS and \%AS at high stretch values. The eccentricity varies between 1.22 and 6 , with a mean value of $2.64 \pm 1.77$ (Fig. 5). 
Table 1. Clinical and demographic patient characteristics. Renal failure defined as calculated glomerular filtration rate according to the Cockcroft-Gault formula $<60 \mathrm{~mL} / \mathrm{min}$.

\begin{tabular}{lcccc}
\hline Patient characteristics & Entire group & $\begin{array}{c}\text { No-SB predilatation } \\
(\mathbf{n}=\mathbf{3 1})\end{array}$ & $\begin{array}{c}\text { SB predilatation } \\
(\mathbf{n}=18)\end{array}$ & $\mathbf{P}$ \\
\hline Age [years] & $66 \pm 11$ & $64 \pm 12$ & $65 \pm 11$ & 0.611 \\
Sex - males & $32(66 \%)$ & $21(77 \%)$ & $11(61 \%)$ & 0.232 \\
Hypertension & $49(100 \%)$ & $31(100 \%)$ & $18(100 \%)$ & 1 \\
Hyperlipidemia & $44(90 \%)$ & $28(84 \%)$ & $16(89 \%)$ & 0.637 \\
Diabetes & $23(47 \%)$ & $14(45 \%)$ & $9(50 \%)$ & 0.750 \\
Renal failure & $21(43 \%)$ & $13(39 \%)$ & $8(44 \%)$ & 0.562 \\
Carotid artery disease & $7(14 \%)$ & $4(12 \%)$ & $3(17 \%)$ & 0.336 \\
Smoking & $30(61 \%)$ & $19(63 \%)$ & $11(61 \%)$ & 0.891 \\
Chronic lung disease & $6(12 \%)$ & $2(6 \%)$ & $4(22 \%)$ & 0.109 \\
Previous myocardial infarction & $12(24 \%)$ & $7(23 \%)$ & $5(28 \%)$ & 0.749 \\
Previous PCl & $27(55 \%)$ & $16(52 \%)$ & $11(61 \%)$ & 0.529 \\
\hline
\end{tabular}

$\mathrm{PCl}$ - percutaneous coronary intervention; SB — side branch

Since all formulas for calculated long axis ostial elliptical diameter give very close results (excluding QO2 formula), it was decided to use only the Ramanujan formula in the present calculations as it gives closest values to a mean value of all formulas for the derived parameters.

\section{Clinical, angiographic}

and procedural characteristics

A total of 49 patients were included - all with stable angina or with recent onset of unstable angina, but with negative troponin. All patients had a significant $(<0.80)$ FFR in MV with or without significant FFR in side branches. The dominantly treated vessel was left anterior descending artery $(\mathrm{n}=42,86 \%)$ with diagonal branches, and the rest of the cases were circumflex artery with marginal branches $(\mathrm{n}=5,10 \%)$ and right posterior descending artery with its posterolateral branches. The SB was predilated in $37 \%$ of the cases, mainly because of angiographically appearing high-grade ostial stenosis. In all patients, the FFR in MV was $\leq 0.80$, and the initial FFR value was $\leq 0.80$ in 26 (53\%) side branches. Eighteen side branches remained with FFR $\leq 0.80$ after stenting, 8 of which were significantly obstructed (based on FFR) after stenting were not, 9 (18\%) new branches became significantly stenosed and the rest, 14 (29\%) remained insignificantly stenosed before and after stenting in MV. Only one patient had SB predilated despite non-significant FFR initial value. Interestingly, 10 (20\%) patients, despite SB balloon dilatation, the stenosis remained significant after stenting (Table 1).
Relations with FFR measurements and ostial area stenosis: as mentioned in the Methods section, ostial area stenosis at SB ostium after stenting was calculated by using three groups.

- Group 1: Circular ostial shape at SB ostium after MV stenting was assumed. This is a standard estimation of $\mathrm{SB}$ ostial stenosis severity (circular area stenosis - ASc).

- Group 2: Elliptical ostial shape at SB ostium assumed after MV stenting - calculated with SB reference diameter, taken into account for long axis ellipse calculation according to formula 5 (Ramanujan) - ASds.

- Group 3: Elliptical ostial shape at SB ostium assumed after MV stenting, calculated with minimal lumen diameter at SB ostium before stenting, considered for long axis ellipse calculation (instead of ds, SBRVD, an ostial minimal lumen diameter at the ostium of SB is used) according to formula 5 (Ramanujan) - ASmld. Each comparison was made for the entire group, and in groups with and without SB predilatation. In the group with SB predilatation, there were better correlations with FFR values with ASds (as the ostial area after balloon predilatation is assumed circular and closer to the reference vessel diameter and consequent transition circular-to-elliptic will operate according to a circular shape of the reference vessel). In group without SB predilataion, FFR will be better correlated with ASmld. In the last group, it was assumed that the circular ostial shape of initial SB minimal ostial diameter, which deforms to ellipse after stenting. In general, there was a significant change in 
Table 2. Angiographic and procedural characteristics of patients.

\begin{tabular}{lcccc}
\hline & Entire group & No-SB predilatation & SB predilatation & P \\
\hline MV RVD [mm] & $3.36 \pm 0.29$ & $3.38 \pm 0.27$ & $3.28 \pm 0.26$ & 0.189 \\
MV \%DS [\%] & $51 \pm 22$ & $60 \pm 21$ & $58 \pm 20$ & 0.766 \\
MV \%DS [\%], final & $3 \pm 9$ & $2 \pm 5$ & $3 \pm 9$ & 0.614 \\
MB RVD [mm] & $2.97 \pm 0.21$ & $2.99 \pm 0.25$ & $2.96 \pm 0.18$ & 0.585 \\
MB \%DS [\%] & $61 \pm 18$ & $67 \pm 13$ & $71 \pm 11$ & 0.220 \\
MB \%DS [\%], final & $6 \pm 16$ & $2 \pm 6$ & $3 \pm 1$ & 0.308 \\
SB RVD [mm] & $2.51 \pm 0.27$ & $2.45 \pm 0.29$ & $2.53 \pm 0.26$ & 0.330 \\
SB \%DS [\%] & $52 \pm 24$ & $46 \pm 24$ & $71 \pm 14$ & 0.001 \\
SB \%DS [\%], post stenting & $67 \pm 27$ & $65 \pm 27$ & $82 \pm 15$ & 0.008 \\
SB \%DS [\%], final & $39 \pm 33$ & $42 \pm 33$ & $31 \pm 36$ & 0.299 \\
SYNTAX score & $12 \pm 4$ & $12 \pm 4$ & $14 \pm 4$ & 0.231 \\
Multi-vessel disease & $21(43 \%)$ & $14(45 \%)$ & $7(39 \%)$ & 0.677 \\
Stent diameter [mm] & $2.97 \pm 0.36$ & $2.97 \pm 0.35$ & $2.96 \pm 0.38$ & 0.930 \\
Total stent length [mm] & $46 \pm 22$ & $45 \pm 24$ & $48 \pm 17$ & 0.629 \\
Stent implantation pressure [atm] & $13 \pm 1$ & $13 \pm 2$ & $13 \pm 3$ & 0.318 \\
FFR-MB, before stenting & $76 \pm 9$ & $70 \pm 10$ & $71 \pm 6$ & $76 \pm 11$ \\
FFR-SB, before stenting & $82 \pm 9$ & $81 \pm 9$ & $72 \pm 13$ & 0.733 \\
FFR-SB, after stenting & $78 \pm 13$ & $90 \pm 4$ & $90 \pm 4$ & 0.100 \\
FFR-MB, final & $88 \pm 5$ & $89 \pm 5$ & $87 \pm 6$ & 0.024 \\
FFR-SB, final & $90 \pm 4$ & 0.895 \\
\hline
\end{tabular}

$\mathrm{MB}$ - main branch; MV - main vessel; SB — side branch; RVD - reference vessel diameter; mm - proximal MV reference vessel diameter in $\mathrm{mm}$; MV \%DS [\%] — proximal MV percentage diameter stenosis; RVD [mm] — distal main branch reference vessel diameter in mm; MB \%DS [\%] - distal main branch percentage diameter stenosis; SB RVD [mm] — SB reference vessel diameter in mm; SB \%DS [\%] - SB percentage diameter stenosis before stenting; SB \%DS [\%], post stenting - SB percentage diameter stenosis immediately after stent implantation in MV; SB \%DS [\%], final - final SB percentage diameter stenosis, after PCl completion; SYNTAX score - SYNergy between $\mathrm{PCl}$ with TAXus and cardiac surgery; FFR - fractional flow reserve

all calculated parameters for area stenosis after stenting in comparison with area stenosis before stenting: ASc initial vs. ASc poststenting - $93 \% \pm$ $\pm 7 \%$ vs. $81 \% \pm 29 \%, \mathrm{p}=0.002$; ASc initial vs. ASmld poststenting $-93 \% \pm 7 \%$ vs. $77 \% \pm$ $\pm 24 \%, \mathrm{p}<0.001$; ASc initial vs. ASds poststenting $-93 \% \pm 7 \%$ vs. $56 \% \pm 28 \%, \mathrm{p}<0.001$. These imply a significant decrease in area stenosis after stenting, which is in contrast with an increase in diameter stenosis after stenting from $52 \% \pm 24 \%$ before stenting implantation vs. $67 \% \pm 27 \%$ after stenting (Table 2).

The correlation coefficients for the entire group with FFR values after stent implantation were: $r=-0.326, p=0.025$ for ASc; $r=-0.416, p=0.004$ for ASds; $r=-0.511, p<0.001$ for ASmld. Interestingly, when analyzed separately, there was no significant correlation in the group with $\mathrm{SB}$ predilatation between FFR and calculated area stenosis by any method. In contrast, there was a significant correlation between FFR after stenting and SB ostial area stenosis in the group without SB predilatation - with the highest cor- relation between ASmld $(\mathrm{r}=-0.495, \mathrm{p}=0.006)$ and non-significant with ASc $(\mathrm{r}=-0.302$, $\mathrm{p}=0.099$ ). In general, the area stenosis was significantly larger when estimated by the circle formula, than with the ellipse formula - ASc vs. ASds with $25 \% \pm 13 \%, \mathrm{p}<0.001$, ASc vs. ASmld with $9 \% \pm 10 \%, p<0.001$. The differences were also significant in groups with and without SB predilatation.

When compared in groups with $(\mathrm{n}=24,49 \%)$ and without $\mathrm{SB} F F R \leq 0.80$, there was no significant difference in calculated circular shape ostial areas stenosis, ASc (FFR $\leq 0.80$ vs. FFR $>0.80-94 \%$ $\pm 8 \%$ vs. $82 \% \pm 25 \%, \mathrm{p}=0.098)$, but both calculated elliptical area stenoses were significantly different - ASmld (FFR $\leq 0.80$ vs. FFR $>0.80$ : $88 \% \pm 9 \%$ vs. $76 \% \pm 13 \%, \mathrm{p}=0.008)$ and ASds $($ FFR $\leq 0.80$ vs. FFR $>0.80: 68 \% \pm 20 \%$ vs. $52 \% \pm$ $\pm 23 \%, \mathrm{p}=0.033)$. On receiver-operating curve (ROC) analysis a cut-off value for identification of FFR $\leq 0.80$ was found - for ASmld $>83 \%$, ASds $>62 \%$, ASc $>93 \%$, with corresponding sensitivity analysis presented in Table 3 . 


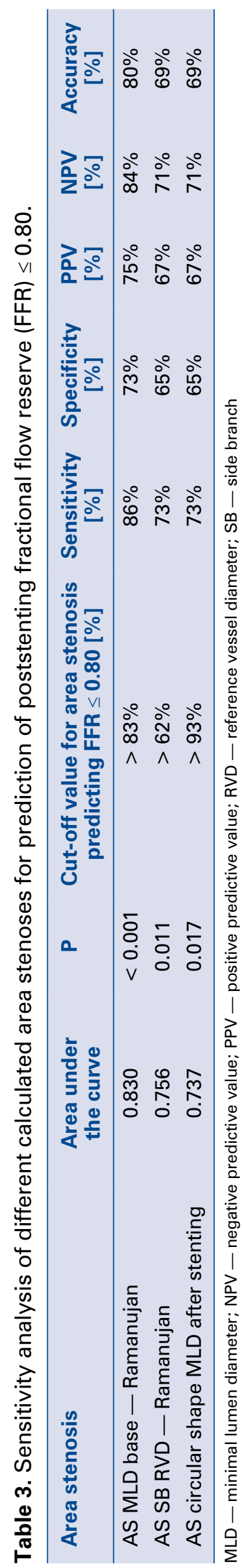

The ostial eccentricity (for ASmld $5.06 \pm 4.66$ vs. $2.89 \pm 1.43$, for FFR $\leq 0.80$ vs. FFR $>0.80$ accordingly, $\mathrm{p}=0.093$ ) despite being numerically higher in the group with lower FFR, and was not statistically different. The last observation suggests, that lower FFR values are associated with greatest ostial elliptic deformations, probably in cooperation with carina displacement. Comparing further, the four groups depended on FFR changes which were $(<$ or $>0.80)$, as described above - negative FFR before and after stenting (group 0), newly appearing significant SB FFR $<0.80$ (group 1), those with significant SB FFR before and after stenting (group 2) and finally patients with initially significant SB FFR, but non-significant after stenting (group 3). The analysis of variance revealed statistically significant differences between groups (ANOVA, $\mathrm{p}=0.001$ for ASmld, $\mathrm{p}=0.043$ for ASds, $\mathrm{p}=0.375$ for $\mathrm{ASc})$. The Bonferroni correction post-hoc multiple comparison test demonstrated highly significant differences between groups 2 and 0 for ASmld $(92 \% \pm 7 \%$ vs. $68 \% \pm 23 \%$, $\mathrm{p}=0.001)$ and borderline differences between groups 1 and 0 for ASmld $(87 \% \pm 12 \%$ vs. $68 \% \pm$ $\pm 23 \%, \mathrm{p}=0.050)$. This suggests a pattern of change in FFR values (i.e., change in ostial area stenosis) regardless of its absolute values may influence FFR values after stenting.

On univariate regression analysis, significant associations of SBFFR $\leq 0.80$ after stenting MV were: history of ST-segment elevation myocardial infarction in the past, presence of carotid artery disease, SYNTAX score, basic systolic blood pressure at the beginning of the procedure, stent diameter, $\mathrm{SB}$ predilatation, SB percentage diameter stenosis before and after stenting, minimal lumen diameter at SB ostium at baseline and after stenting, ASmld, ASds, ASc. A ROC analysis was performed to identify cut-off values for identification of FFR less than 0.80 about area stenosis calculated values (Table 3). The ASmld $>83 \%$, ASds $>62 \%$ and ASc $>93 \%$ were also significantly associated with cut-off FFR on univariate regression analysis. On multivariate logistic regression analysis ASmld $>83 \%$, but neither circular area stenosis values nor continuous parameter was independently associated with FFR $\leq 0.80$ (OR 7.143, CI 1.006-50.000; Negelkerke R square 0.477, $\mathrm{p}=0.002$, Hosmer and Lemeshov $\mathrm{p}=0.427$ ).

\section{Discussion}

Over a decade ago, the deformation of the circular opening before stenting to elliptical opening 
after stenting as a mechanism for SB compromise based on theoretical assumptions and observations from phantom elastic models of coronary bifurcations was proposed [2, 3]. In recent years, these theoretical and experimental observations were confirmed in optical coherence tomography imaging of coronary bifurcations after stenting MV $[5,6]$. The current study provides a quantitative basis for area stenosis calculation, based on angiography data. The formulas used were adopted from the literature [8-15] and constrained based on constant vessel circumference assumption. A formula from Ramanujan was used [8, 9, 13] which provides values closest to a mean from all other formulas.

The area stenosis calculated based on elliptical assumptions provides much more physiological ostial area stenosis in better agreement with experimental observation on flow limitations caused from stenosis $[16,17]$. This is more important in less severe stenoses ( $<70 \%$ area stenosis), where differences in areas are larger, while in more severe stenosis ( $>70 \%$ area stenosis) the shape of the ostium does not seem to be so important and the values of area stenosis are more circular, no matter which formula is used. It should be noted that the present calculations are based on the assumption of the ability of ostium of SB to deform freely, quantitatively expressed by a stretching coefficient $\mathrm{k}$. In reality, the presence of plaque with fibrous content and calcium can preclude these deformations [18].

To provide a better association between area stenosis and functional stenosis, calculated data was compared with experimental data for functional stenosis (i.e., FFR). The area stenosis in patients from the FIESTA study was calculated using formulas based on elliptical shape correlates better with parameter for functional stenosis, such as FFR, than the area stenosis calculated based on circle form of the ostium ( $r=-0.326$ for ASc; $r=$ -0.416 for ASds; $r=-0.511$ for ASmld). Moreover, the area stenosis based on elliptical shape (ASmld) has significantly better accuracy for identification of significant FFR after stenting, than area stenosis, calculated based on circular ostial shape. For the first time, a practical form for calculation of ellipse type of opening was provided herein, based only on angiographic data. These formulas can be implemented in future with software programs for automatic analysis. It can be speculated that elliptical ostial shaping is a final common pathway that occurs at the ostium after main vessel stenting. Elliptical stretch and deformation could occur and can explain side branch ostial stenosis (even high-grade) at $90^{\circ}$ occurring branches from the main vessel, where carina shifting is theoretically impossible.

In accordance with the assumptions for initial circular side branch minimal lumen diameter, which transforms to ellipse, are data from the literature, demonstrating that almost $90 \%$ of bifurcations have circular ostia [5, 6]. Why the ASmld formula performs best in prediction of significant FFR after main vessel stenting? The most plausible reason is that it relays on three different parameters (side branch MLD before and after stenting and $\mathrm{SB}$ reference diameter), while ASds and ASc relay only on two parameters - SBMLD after stenting and SBRVD. Thus, ASmld incorporates the basic information of lesion flow limiting capacity, not only information obtained after stenting.

The areas at the ostium calculated in the present study are considerably smaller than those reported in the literature $[5,6,19,20]$. This may be explained by differences in imaging methods (angiography, optical coherence tomography, or intravascular ultrasound). Optical coherence tomography visualizes the shape and size of SB, while the minimum ostial area (calculated based on angiographic data) can appear at a distance from the SB opening, because of invagination of the side vessel wall - see Figure 1 from reference no. 6 . One possible explanation is a difference in patient populations - the present group has larger SB reference diameters than those previously reported (2.51 mm vs. $2.0-2.2 \mathrm{~mm}$ in other studies). All our patients have significant MV FFR values before interventions and practically half of the current patients had functionally true bifurcations. This percentage is larger than typically reported (around $30 \%$ or less).

\section{Limitations of the study}

The present study has several limitations. First, it did not consider the other two mechanisms of SB compromise - carina displacement and plaque shift. The theoretical data and crosscalculated parameters assuming elliptical ostial deformation of SB ostium after stenting however, correlate very well with the parameter of physiological severity, namely FFR. It must be pointed out, that carina displacement is one of the suggested mechanisms causing ellipse formation at SB ostium. This may explain the good correlations observed in the present study. Further research is needed to implement carina and plaque shifts in the model to better predict the observed changes. Second, only angiographic analysis and measure- 
ments of vessel sizes was performed. This is subject to a significant inaccuracy and variation. Three-dimensional optical coherence tomography imaging might have offered better visualization of the elliptic deformation of the SB ostium. However, such elliptic deformation has already been demonstrated by others [5, 6]. Third, the group of patients is relatively small. Given that half of angiographically significant coronary bifurcation stenosis are functionally insignificant by means of FFR values, however, it becomes rather impractical to find many appropriate patients for such a study [21].

\section{Conclusions}

The elliptical ostial transformation of side branches after MV stenting of coronary bifurcation is a possible mechanism for SB compromise. The ostial area stenosis, calculated based on this assumption correlates better with the physiological parameter of lesion severity, i.e. FFR, compared to area stenosis calculated based on the traditional circular formula.

\section{Conflict of interest: None declared}

\section{References}

1. Lassen JF, Holm NR, Banning A, et al. Percutaneous coronary intervention for coronary bifurcation disease: 11th consensus document from the European Bifurcation Club. EuroIntervention. 2016; 12(1): 38-46, doi: 10.4244/EIJV12I1A7, indexed in Pubmed: 27173860.

2. Vassilev D, Gil RJ. Relative dependence of diameters of branches in coronary bifurcations after stent implantation in main vessel-importance of carina position. Kardiol Pol. 2008; 66(4): 371-379, indexed in Pubmed: 18473265.

3. Vassilev D, Gil RJ. Changes in coronary bifurcations after stent placement in the main vessel and balloon opening of stent cells: theory and practical verification on a bench-test model. J Geriatr Cardiol. 2008; 5: 43-49.

4. Vassilev D, Gil R. Clinical verification of a theory for predicting side branch stenosis after main vessel stenting in coronary bifurcation lesions. J Interv Cardiol. 2008; 21(6): 493-503, doi: 10.1111/j.15408183.2008.00400.x, indexed in Pubmed: 18973506.

5. Cho S, Kim JS, Ha J, et al. Three-Dimensional optical coherence tomographic analysis of eccentric morphology of the jailed sidebranch ostium in coronary bifurcation lesions. Can J Cardiol. 2016; 32(2): 234-239, doi: 10.1016/j.cjca.2015.06.001, indexed in Pubmed: 26341304.

6. Yang PS, Ha J, Kim JS, et al. Eccentric morphology of jailed side-branch ostium after stent crossover in coronary bifurcation lesions: a three-dimensional optical coherence tomographic analysis. J Cardiol. 2015; 65(4): 305-310, doi: 10.1016/j. jjcc.2014.12.004, indexed in Pubmed: 25601268.

7. Kolh P, Windecker S, Alfonso F, et al. European Society of Cardiology Committee for Practice Guidelines, EACTS Clinical Guidelines Committee, Task Force on Myocardial Revascularization of the European Society of Cardiology and the European As- sociation for Cardio-Thoracic Surgery, European Association of Percutaneous Cardiovascular Interventions, Authors/Task Force members. 2014 ESC/EACTS Guidelines on myocardial revascularization: The Task Force on Myocardial Revascularization of the European Society of Cardiology (ESC) and the European Association for Cardio-Thoracic Surgery (EACTS)Developed with the special contribution of the European Association of Percutaneous Cardiovascular Interventions (EAPCI). Eur Heart J. 2014; 35(37): 2541-2619, doi: 10.1093/eurheartj/ehu278, indexed in Pubmed: 25173339.

8. Sýkora S. Approximations of Ellipse Perimeters and of the Complete Elliptic Integral E(x). Review of known formulae DOI:10.3247/SL1Math05.004. http://www.ebyte.it/library/docs/ math05a/EllipsePerimeterApprox05.html.

9. Sýkora S. Advances in Approximations of Ellipse Perimeters and of the Complete Elliptic Integral. DOI: 10.3247/SL2Math07.001. http://www.ebyte.it/library/docs/math07/EllipsePerimeterApprox07.html.

10. Almkvist G, Berndt B. Gauss, Landen, Ramanujan, the Arithmetic-Geometric Mean, Ellipses, $\varpi$, and the Ladies Diary. The American Mathematical Monthly. 2018; 95(7): 585-608, doi: 10.1080/00029890.1988.11972055.

11. Hudson RG, Lipka J, A manual of mathematics, 1st Edition 1917.

12. Lockwood EH. Length of ellipse. The Mathematical Gazette. 1932; 16(220): 269-270, doi: 10.2307/3605929.

13. Gérard P. Michon Perimeter of an Ellipse. http://www.numericana.com/answer/ellipse.htm.

14. Latka F., Collection of Mathematical Formulae, Hungarian Edition, Budapest 1985.

15. Barnard R, Pearce K, Schovanec L. Inequalities for the Perimeter of an Ellipse. Journal of Mathematical Analysis and Applications. 2001; 260(2): 295-306, doi: 10.1006/jmaa.2000.7128.

16. Gould KL, Johnson NP. Physiologic stenosis severity, binary thinking, revascularization, and "hidden reality". Circ Cardiovasc Imaging. 2015; 8(1), doi: 10.1161/CIRCIMAGING.114.002970, indexed in Pubmed: 25596144.

17. Gould KL, Johnson NP, Bateman TM, et al. Anatomic versus physiologic assessment of coronary artery disease. Role of coronary flow reserve, fractional flow reserve, and positron emission tomography imaging in revascularization decision-making. J Am Coll Cardiol. 2013; 62(18): 1639-1653, doi: 10.1016/j. jacc.2013.07.076, indexed in Pubmed: 23954338.

18. Johnson PM, Madamanchi C, Sharalaya ZM, et al. Angiographic severity does not correlate with fractional flow reserve in heavily calcified coronary arteries. Catheter Cardiovasc Interv. 2017; 89(2): 226-232, doi: 10.1002/ccd.26635, indexed in Pubmed: 27465149 .

19. Ha J, Kim JS, Mintz GS, et al. 3D OCT versus FFR for jailed side-branch ostial stenoses. JACC Cardiovasc Imaging. 2014; 7(2): 204-205, doi: 10.1016/j.jcmg.2013.06.011, indexed in Pubmed: 24524746 .

20. Kang SJ, Kim WJ, Lee JY, et al. Hemodynamic impact of changes in bifurcation geometry after single-stent cross-over technique assessed by intravascular ultrasound and fractional flow reserve. Catheter Cardiovasc Interv. 2013; 82(7): 1075-1082, doi: 10.1002/ccd.24956, indexed in Pubmed: 23592548.

21. Vassilev D, Dosev L, Collet C, et al. Intracoronary electrocardiogram to guide percutaneous interventions in coronary bifurcations - a proof of concept: the FIESTA (Ffr vs. IcEcgSTA) study. EuroIntervention. 2018; 14(5): e530-e537, doi: 10.4244/ /eij-d-17-00189. 